\title{
Co-overexpression of bacterial GroESL chaperonins partly overcomes non-productive folding and tetramer assembly of E. coli-expressed human medium-chain acyl-CoA dehydrogenase (MCAD) carrying the prevalent disease-causing K304E mutation
}

\author{
Peter Bross a , Brage S. Andresen a , Vibeke Winter a , Franz Kräutle ${ }^{c}$, \\ Thomas G. Jensen ${ }^{b}$, Andreas Nandy ${ }^{c}$, Steen Kølvraa ${ }^{b}$, Sandro Ghisla ${ }^{c}$, \\ Lars Bolund ${ }^{\mathrm{b}}$ and Niels Gregersen ${ }^{\mathrm{a}}$ \\ ${ }^{a}$ Molecular Genetic Laboratory, Dept. of Clinical Chemistry, Arhus Kommunehospital and Skejby Sygehus, Arhus (Denmark), \\ ${ }^{b}$ Institute of Human Genetics, Unicersity of Arhus, Arhus (Denmark) and c Faculty for Biology, University of Konstanz, \\ Konstanz (Germany)
}

Key words: MCAD deficiency; GroE; Chaperonin; Aggregation; Folding; Oligomer assembly

\begin{abstract}
The influence of co-overexpression of the bacterial chaperonins GroEL and GroES on solubility, tetramer formation and enzyme activity of three variants of heterologously-expressed human medium-chain acyl-CoA dehydrogenase (MCAD) was analysed in order to investigate the molecular mechanism underlying MCAD deficiency caused by the prevalent K304E mutation. Depending on which of the three amino acids - lysine (wild-type), glutamic acid (K304E) or glutamine (K304Q) are present at position 304 of the mature polypeptide, three different patterns were observed in our assay system: (i) solubility, tetramer formation and yicld of enzyme activity of wild-type MCAD is largely independent of GroESL co-overexpression; (ii) the larger part of the K304Q mutant is insoluble without and solubility is enhanced with GroESL co-overexpression; solubility correlates with the amount of tetramer detected and the enzyme activity measured as observed for the wild-type protein. (iii) Solubility of the K304E mutant is in a similar fashion GroESL responsive as the $\mathrm{K} 304 \mathrm{Q}$ mutant, but the amount of tetramer observed and the enzyme activity measured do not correlate with the amount of soluble K304E MCAD protein detected in Western blotting. In a first attempt to estimate the specific activity, we show that tetrameric K304E and K304Q mutant MCAD display a specific activity in the range of the wild-type enzyme. Taken together, our results strongly suggest, that the K304E mutation primarily impairs the rate of folding and subunit assembly. Based on the data presented, we propose that lysine-304 is important for the folding pathway and that an exchange of this amino acid both to glutamine or glutamic acid leads to an increased tendency to misfold/aggregate. Furthermore, exchange of lysine-304 with an amino acid with negative charge at position 304 (glutamic acid) but not with a neutral charge (glutamine) negatively affects conversion to active tetramers. A possible explanation for this latter effect - charge repulsion upon subunit docking - is discussed.
\end{abstract}

\section{Introduction}

Medium-chain acyl-CoA dehydrogenase (MCAD; EC 1.3.99.3) is one of four evolutionarily related, chain length specific enzymes which catalyze the first step in mitochondrial $\beta$-oxidation of straight-chain fatty acids

Correspondence to: P. Bross, Molecular Genetic Laboratory, Department of Clinical Chemistry, Skejby Sygehus, DK-8200 Århus N, Denmark.

Abbreviations: IPTG, isopropyl- $\beta$-D-thiogalactopyranoside; MCAD, medium-chain acyl-CoA dehydrogenase; PAGE, polyacrylamide gel electrophoresis; PCR, polymerase chain reaction; PMSF, phenylmethylsulfonyl fluoride. in mammals [1-3]. MCAD protein is nucleus encoded and transported into the mitochondrial matrix where it is processed and folds to the tetrameric flavin containing native enzyme. MCAD deficiency is an autosomal recessive genetic disease which, in the majority of cases (approx. 90\% of the alleles, [4]), is due to a point mutation at position 985 of the cDNA sequence [5-8] causing a lysine to glutamic acid exchange at position 304 (K304E) of the mature protein. The K304E mutant protein is transported into mitochondria and processed correctly [9]. We have shown that, unlike the wildtype enzyme, mature mutant MCAD-expressed in Escherichia coli is to a large extent insoluble leading to formation of insoluble aggregates $[5,10]$. Upon overex- 
pression in COS-7 cells, the K304E mutant has been demonstrated to be unstable and only very small amounts of tetrameric mutant MCAD could be detected [11]. Instability of K304E mutant MCAD has also been observed in patient fibroblasts [12]. Both from expression in E. coli and COS-7 cells it was so far not possible to unambiguously determine whether the K304E mutant MCAD possesses residual activity. Taking the results with expression in E. coli and COS-7 cells together, we speculate, that the decreased amount of functional tetrameric enzyme protein is due to misfolding and/or hampered tetramer assembly.

Chaperonins are a subgroup of the chaperones, which constitute a class of ubiquitous proteins assisting folding and oligomer assembly of proteins in vivo (for recent reviews see Refs. 13 and 14). Chaperonin cpn-60 (GroEL in E. coli) and co-chaperonin cpn-10 (GroES in $E$. coli) cooperate in this process of assisted folding which is considered to proceed in a similar fashion in at least eubacteria and mitochondria. By in vitro refolding studies the binding and release of a series of proteins from different sources to GroEL has been studied and it has been shown that GroEL together with GroES influences the folding process. Further on, co-overexpression studies of the GroES and GroEL chaperonins along with heterologous proteins has indicated that a higher chaperonin level can promote correct folding and oligomer assembly (see Refs. 13 and 14).

To study the phenomenon underlying defective production of active MCAD enzyme in patients with the K304E mutation more closely and in an attempt to produce sufficient amounts of functional K304E mutant enzyme for further studies, we speculated whether increasing the chaperonin level could rescue K304E mutant MCAD tetramers. For this purpose, we cooverexpressed the $E$. coli chaperonins GroES and GroEL along with wild-type or K304E mutant MCAD with the purpose of testing whether this has an influence on folding and oligomer assembly of the mutant enzyme. In order to inspect the importance of the positive charge in the side chain of lysine-304 for folding and oligomer assembly, an artificially constructed variant with glutamine at position 304 (K304Q) was also analyzed in this system.

\section{Materials and Methods}

\section{Strains and plasmids}

Plasmid pBMCK2 ${ }^{-}$, a pBluescriptKS ${ }^{-}$(Stratagene) derivative encoding mature wild-type human MCAD under control of the lac promoter, has been described [9]. For construction of p 985 the Eco RI/BamHI fragment of pBMCK2 $^{-}$encoding the carboxyl-terminal half of MCAD was exchanged with a respective fragment containing the G-985 (K304E) mutation. This fragment was derived from a clone of PCR amplified cDNA (using primers pri(s)-18: 5'-CCGGGGATCCGAGCCAACATGGCAGCGGGG-3' and pri(a) + 1305: 5'-TTTCGGATCCCTGGAGCTGAAACAGTGGCT-3') from a patient. pGroESL was a gift of Dr. A.A. Gatenby and has been described by Goloubinoff et al. [15]. pGroESL carries the GroES and GroEL genes under control of the lac promoter. The plasmid has a p15A replicon which is compatible with plasmids with the pBR322-derived colE1 replicon like pBluescriptKS ${ }^{-}$and carries a chloramphenicol resistance marker. The control plasmid $\mathrm{pCaP}$ was constructed by digestion of plasmid pGroESL with Pst I and subsequent ligation of the largest fragment. By this treatment, 6 Pst I fragments encoding the GroESL genes are removed (nucleotide position 225-1982 of the published sequence [16]). All plasmids were transformed into $E$. coli JM109 [17].

\section{Growth and disruption of cells}

Cells were grown in dYT [18] supplemented with ampicillin and chloramphenicol. Cell density was followed spectrophotometrically. Cells were disrupted with a combined lysozyme/sonication procedure as follows: cells (pellet from $4-10 \mathrm{ml}$ culture) were suspended in $300 \mu \mathrm{l}$ buffer A (50 mM Tris-Cl (pH 8.0), $25 \%$ sucrose, $0.1 \mathrm{mM}$ PMSF, $12 \mu \mathrm{M}$ FAD) at $0^{\circ} \mathrm{C} .60$ $\mu 10.5 \mathrm{M}$ EDTA and after $5 \mathrm{~min}$ at $0^{\circ} \mathrm{C} 60 \mu 1$ lysozyme solution $(2 \mathrm{mg} / \mathrm{ml}$ in $250 \mathrm{mM}$ Tris- $\mathrm{Cl}, \mathrm{pH} 8.0)$ were added. After 5 more minutes $600 \mu \mathrm{l}$ of lysis solution (50 mM Tris-Cl (pH 8.0), $62.5 \mathrm{mM}$ EDTA, 0.1\% Triton $\mathrm{X}-100$ ) was added and the extract sonicated for $30 \mathrm{~s}$ (microtip, 50\% duty cycle) followed by centrifugation at $15000 \times g$ for $30 \mathrm{~min}$. The supernatant was withdrawn and stored at $-20^{\circ} \mathrm{C}$.

\section{Activity measurements}

MCAD activity was determined with the mass spectrometric product formation assay as described by Kølvraa et al. [19] or with the ferricenium assay essentially as described by Lehman et al. [20].

\section{Gel filtration chromatography}

Gel filtration chromatography was carried out with a Pharmacia Superose 12 column using $150 \mathrm{mM}$ potassium phosphate ( $\mathrm{pH} 8.25$ ) supplemented with $1 \mu \mathrm{M}$ FAD as buffer. Flow rate was $0.1-0.15 \mathrm{ml} / \mathrm{min}$ and fractions of $0.5 \mathrm{ml}$ were collected. Absorption at 280 $\mathrm{nm}$ was recorded.

\section{Construction of $K 304 Q$ mutant}

This mutant was constructed by site-directed mutagenesis using PCR according to Kuipers et al. [21]. In brief, single-stranded DNA of plasmid pBMCK2 $2^{-}$was used as template for PCR with a mutagenic primer pri(s)K304Q (5'-GAAATGGCAATGCAGGTTGAA- 
A - Gro E $\quad+$ Gro E
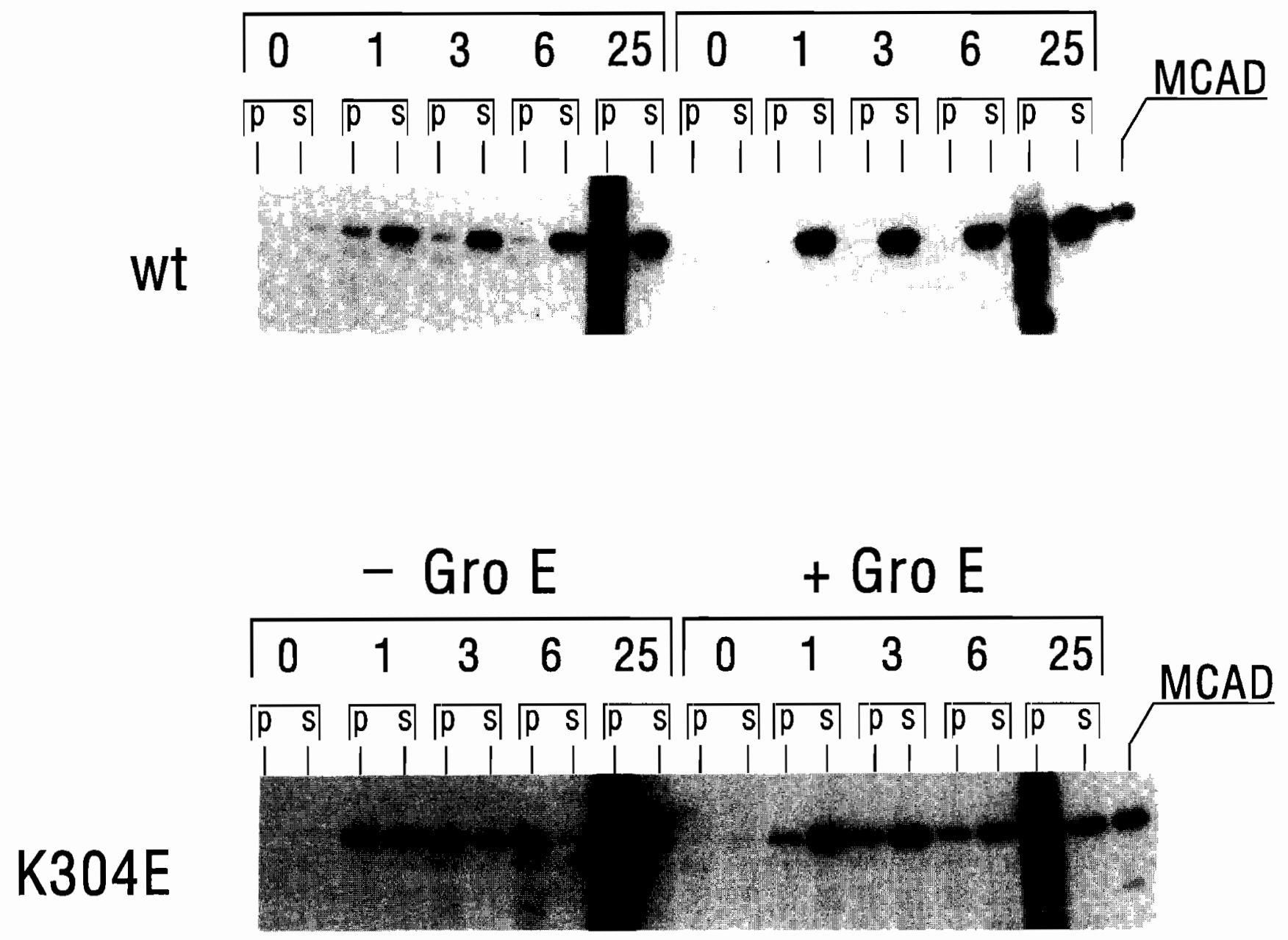

B

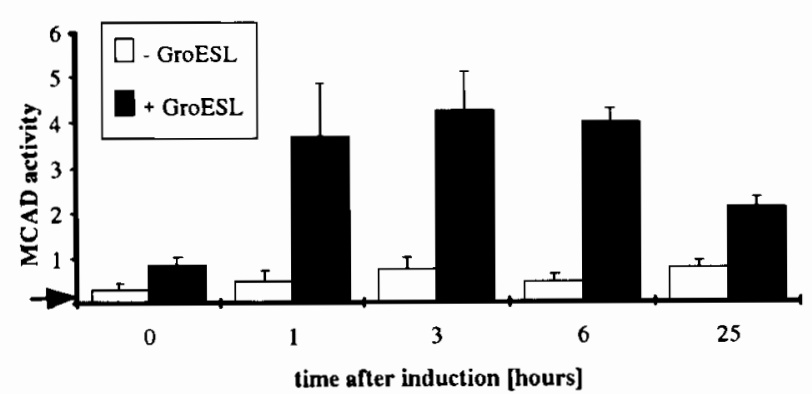

B wild type

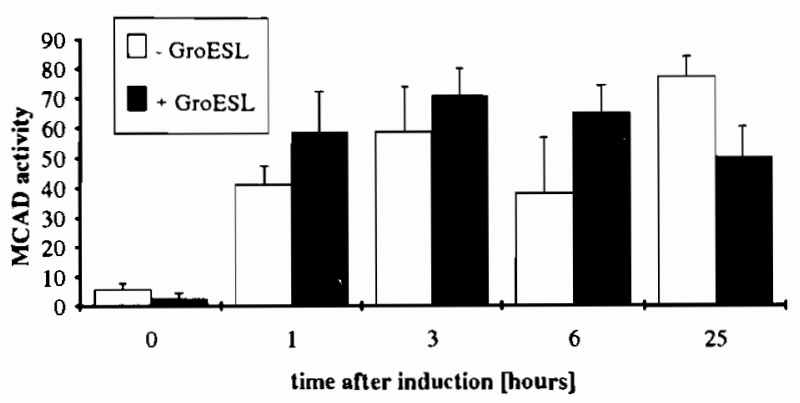

Fig. 1. Influence of GroESL co-overexpression on solubility and enzyme activity of wild-type and K304E mutant MCAD. (A) JM109 cells cotransformed with pBMCK2 ${ }^{-}$encoding mature wild-type (wt) MCAD or p985-41 encoding K304E mutant MCAD and pGroESL ( + GroE) or pCaP ( - GroE), a control plasmid lacking the GroESL genes, were grown to a density of approx. $8 \cdot 10^{8}$ cells $/ \mathrm{ml}$ and expression of both the MCAD and the GroESL genes was induced with $1 \mathrm{mM}$ IPTG. At the time points indicated, samples were taken. The cells were disrupted, separated by centrifugation into pellet $(\mathrm{p})$ and supernatant (s) fractions and subjected to SDS-PAGE followed by Western blotting using anti-MCAD antibodies as described in Materials and Methods. $1.25 \mu \mathrm{g}$ protein of the supernatant fractions and a corresponding aliquot of the pellet fractions were loaded per lane. Purified recombinant MCAD protein was co-electrophoresed as marker. (B) MCAD activity in the supernatant fractions from the above experiment was determined using the product formation assay. MCAD activity is given in $\mu$ mol hydroxy-octanoic acid formed per mg protein and minute $\times 10^{-3}$. The arrow indicates the control level measured in extracts from cells carrying the vector plasmid without an MCAD gene (see text). 
CTAGC-3') and primer pri(a) + 1305 (5'-TTTCGGATCCCTGGAGCTGAAACAGTGGCT-3'). PCR (15 cycles) was performed with a Cetus-Perkin Elmer thermal cycler and the fragment comprising nucleotides 973 to 1322 and an adjacent Bam HI site on the 3 '-end was gel purified and used as primer in a second PCR reaction with single-stranded pBMCK2 ${ }^{-}$ DNA as template and primer pri(s) + $378\left(5^{\prime}\right.$-TTCTTTGGGGCAAATGCCTA-3'). The amplified fragment was gel purified, cleaved with $E c o$ RI at the site within the MCAD cDNA sequence and Bam $\mathrm{HI}$ and ligated into the large fragment of $\mathrm{pBMCK} 2^{-}$which had been cleaved with the same enzymes. After transformation into $E$. coli, 5 clones were sequenced in order to check for introduction of the mutation and PCR errors. One of the desired clones was selected and the plasmid was termed pK304Q.

\section{Gel electrophoresis and Western blotting}

SDS-PAGE was performed using the discontinuous buffer system described by Laemmli [22]. Native PAGE was with $4-15 \%$ Phastgel media and native buffer strips (Pharmacia) as recommended by the supplier. Western blotting was performed with a semi-dry apparatus (Pharmacia) as described by Blake et al. [23]. Anti-pig kidney MCAD antibodies or anti-GroEL antibodies (a gift from Dr. A.A. Gatenby) were used and visualized with phosphatase conjugated secondary antibodies (DAKO) and chemiluminescence detection using AMPPD (Tropix).

\section{Purification of wild-type and mutant MCAD}

Cells from induced cultures transformed with pGroESL and the respective MCAD plasmid were disrupted as described above using $500 \mu \mathrm{l}$ of buffer A and respective volumes of the other solutions for a pellet from $200 \mathrm{ml}$ cells. The supernatant was diluted into $10 \mathrm{ml}$ of potassium-phosphate buffer $(50 \mathrm{mM}$ potassium-phosphate, $\mathrm{pH} 8.25 ; 1 \mathrm{mM}$ EDTA) and subjected to a $1 \mathrm{ml} \mathrm{Hi-Trap}{ }^{\mathrm{TM}}$ Blue column (Pharmacia). The column was washed with 10 vols. of potassiumphosphate buffer and bound proteins were subsequently eluted with $3 \mathrm{ml}$ elution buffer $(50 \mathrm{mM}$ potassium-phosphate, $\mathrm{pH} 8.25 ; 1 \mathrm{mM}$ EDTA; $0.5 \mathrm{M} \mathrm{NaCl}$; $12 \mu \mathrm{M}$ FAD). The eluate was diluted 3-fold with potassium-phosphate buffer to lower the $\mathrm{NaCl}$ concentration, concentrated by ultrafiltration (Centriprep 30, Centricon 30; Amicon) to about $100 \mu \mathrm{l}$ and subjected to Superose 12 gel filtration chromatography as described above. MCAD containing fractions were detected by the MCAD enzyme activity assay using ferricenium as electron acceptor which was performed essentially as described by Lehman et al. [21]. MCAD peak fractions were pooled. The potassium-phosphate concentration was adjusted to $50 \mathrm{mM}, \mathrm{FAD}$ added to $12 \mu \mathrm{M}$ and the volume was reduced to about $60 \mu \mathrm{l}$ by ultrafiltration as above. Finally, glycerol was added to $5 \%$ and the purified enzyme was stored at $4^{\circ} \mathrm{C}$.

\section{Results}

GroESL co-overexpression promotes solubility and yield of active K304E mutant MCAD

As described in a preliminary report [10], overexpression of mature MCAD with the disease causing $\mathrm{K} 304 \mathrm{E}$ mutation in $E$. coli produced misfolded aggregated protein while wild-type MCAD-expressed in the same system folds to active enzyme. As aggregates are thought to derive from partial intracellular denaturation of folding intermediates [24] and chaperonins can prevent folding intermediates from aggregation, we decided to test whether inefficient production of native enzyme could be overcome by excess chaperonin proteins. For this purpose we used the approach described by Goloubinoff et al. [15] to co-overexpress the $E$. coli chaperonins GroES and GroEL. E. coli JM109 carrying both the respective wild-type or mutant MCAD expression plasmid and plasmid pGroESL encoding the groE operon [15] were grown and induced for different time intervals. As control, cells cotransformed with the respective MCAD plasmid and $\mathrm{pCaP}$, a deletion derivative of pGroESL lacking the chaperonin genes, were grown and induced under identical conditions. The cells were disrupted with a combined lysozyme/sonication method to ensure efficient reproducible cell lysis. After subsequent centrifugation, corresponding aliquots of the pellet and supernatant fractions were analyzed by Western blotting (Fig. 1A) in order to determine solubility of the expressed MCAD protein. MCAD enzyme activity in the supernatants was measured with the highly sensitive and specific mass spectrometric assay (Fig. 1B). Wild-type MCAD is mainly detected in the supernatant fractions in the samples until $6 \mathrm{~h}$ after induction and chaperonin cooverexpression has only a minor influence by reducing the small proportion of insoluble MCAD in these samples. After $25 \mathrm{~h}$ induction, wild-type MCAD is found both in the supernatant and the pellet and the pellet contains large amounts of immunoreactive material which smears over a broad molecular weight range.

The activity values parallel the amount of soluble wild-type MCAD protein observed in western blotting. There is a small increase in activity in the samples with GroESL co-overexpression until $6 \mathrm{~h}$ after induction. This might reflect the transformation into active protein of the small proportion of insoluble MCAD detected in the pellet fractions without GroESL co-overexpression.

As far as K304E MCAD is concerned, there is a considerable amount of soluble protein detectable in the supernatant in the sample after $1 \mathrm{~h}$ induction without GroESL co-overexpression. The relative 
amount of MCAD protein detected in the pellet compared to that in the supernatant increases with longer induction times. The total amount of MCAD protein detected is comparable to that of the wild-type protein indicating that the mutant protein is not markedly more unstable.

In all samples without GroESL co-overexpression, also in those with a considerable amount of soluble $\mathrm{K} 304 \mathrm{E}$ protein, MCAD activity is only slightly higher than background. As background, the values from control inductions of cells transformed with the vector plasmid (pBluescriptKS ${ }^{-}$) and pGroESL or pCaP were taken. The control values were constant throughout induction and the mean is indicated by the arrow in Fig. 1B.

Strikingly, co-overexpression of GroESL increases the amount of soluble K304E mutant protein until $6 \mathrm{~h}$ after induction. The partition between pellet and supernatant is reversed as compared to the samples without GroESL co-overexpression. This demonstrates, that GroESL increases solubility of the mutant protein. Furthermore, there is a factor of about 5-7-times more
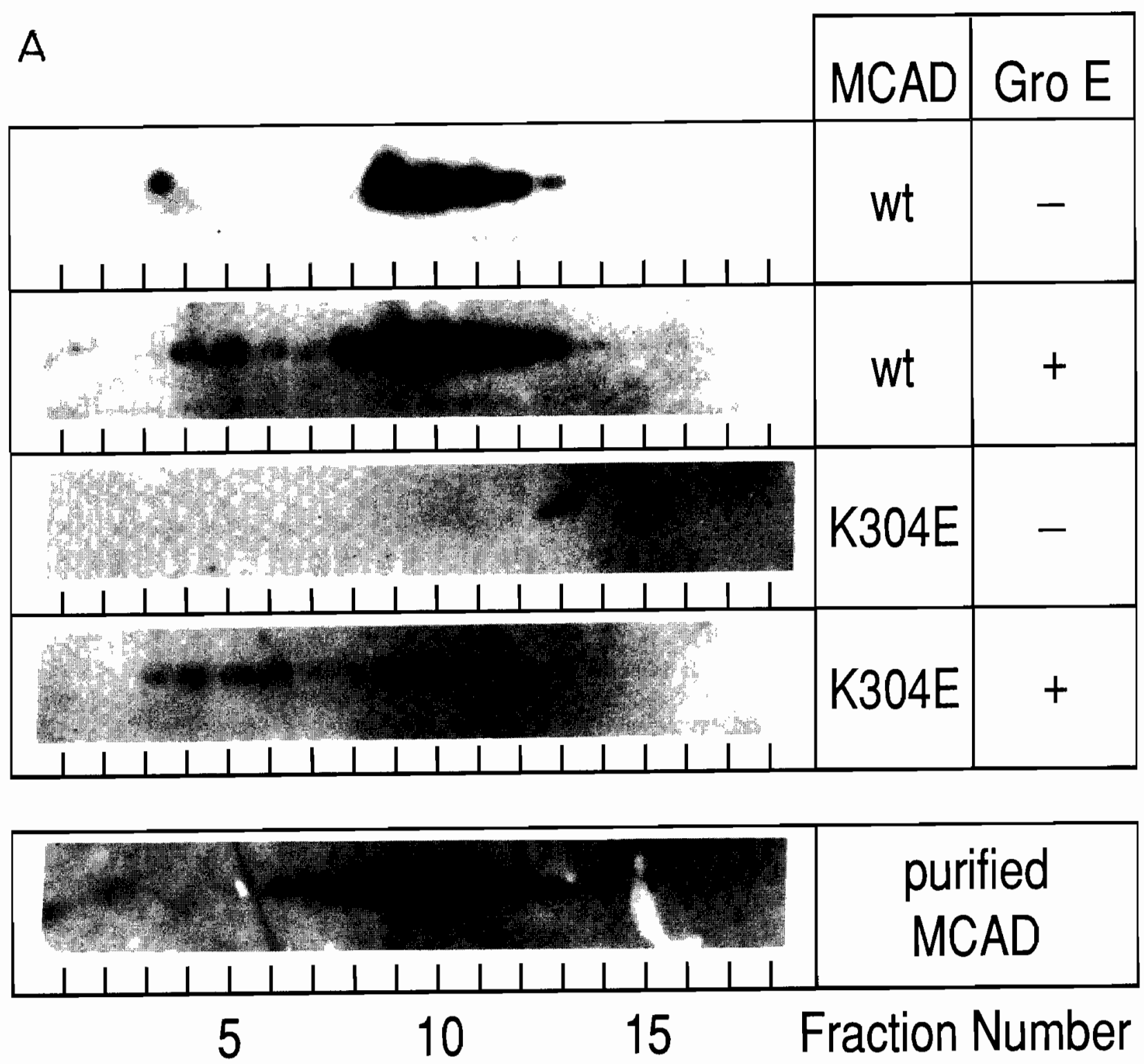

Fig. 2. (A) Gel chromatographic sizing analysis of wild-type and K304E mutant MCAD. Soluble proteins from the $3 \mathrm{~h}$ induction samples in Fig. 1 of wild-type (wt) or K304E MCAD without ( - GroE) or with GroESL ( + GroE) co-overexpression were subjected to Superose-12 gel filtration chromatography. Aliquots of the collected fractions (à $0.5 \mathrm{ml}$ ) were analyzed for MCAD contents by Western blotting using anti-MCAD antibodies. As marker, purified recombinant MCAD was analyzed the same way. (B) MCAD activity (left axis) in fractions from gel filtration chromatography of the extract from cells co-overexpressing GroESL and K304E MCAD was measured with the product formation assay. The corresponding Western blot was scanned and relative scan values are plotted on the right axis. 


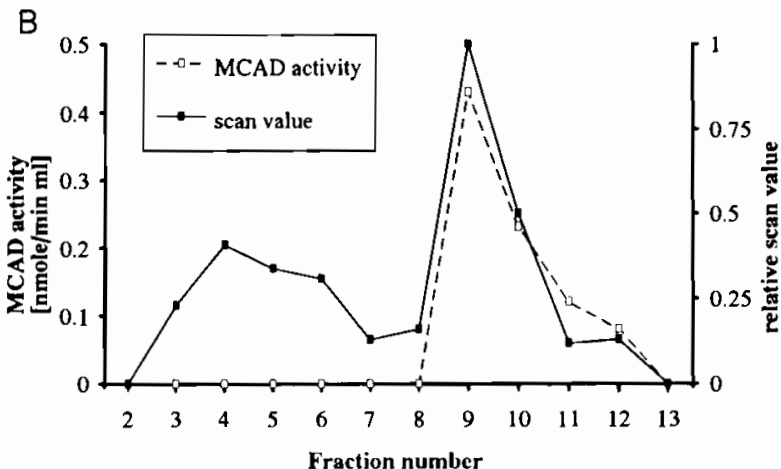

Fig. 2 (continued).

MCAD enzyme activity present in the extracts co-overexpressing K304E mutant MCAD and the chaperonin genes. Like for the wild-type, the activity values peak at $3 \mathrm{~h}$ after induction. It should be stressed, that clearly less MCAD activity is detected for the K304E mutant enzyme as compared to the wild-type (a factor of 16-18), while the difference in the amount of soluble protein in the supernatants with GroESL co-overexpression after 1 to $6 \mathrm{~h}$ induction is not that striking (compare Fig. 1). This was further substantiated by electrophoresing all supernatants on the same gel followed by western blotting and scanning of the MCAD bands (data not shown).

\section{Oligomer assembly of wild-type and mutant MCAD}

In order to analyze the quaternary structure of expressed soluble wild-type and K304E MCAD, gel filtration chromatography of the $3 \mathrm{~h}$ supernatants from the above induction experiment was performed, and the MCAD contents in the column fractions were

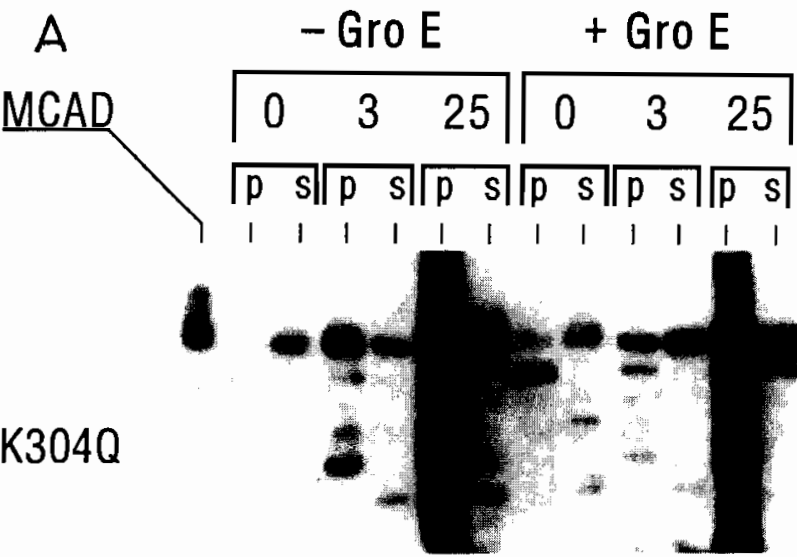

B

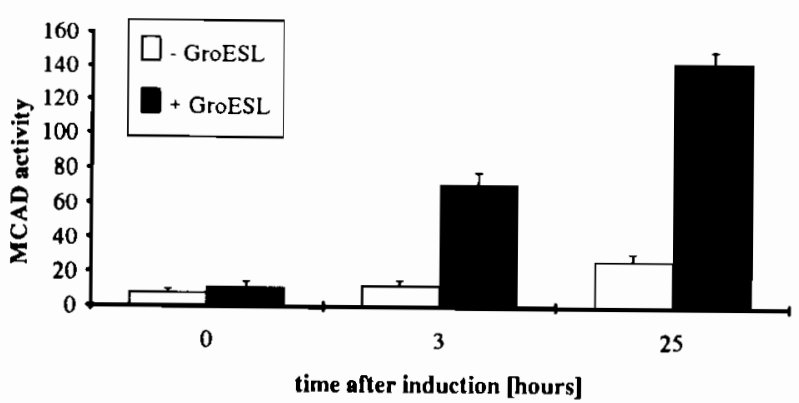

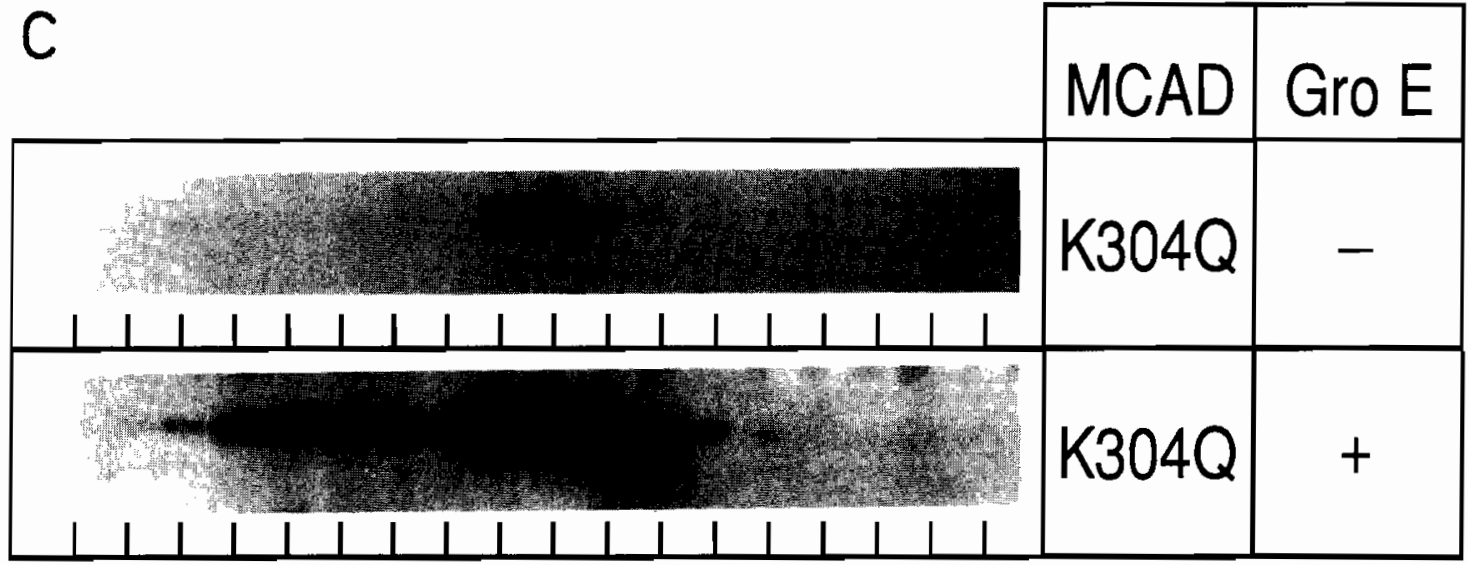

$5 \quad 10 \quad 15 \quad$ Fraction Number

Fig. 3. Solubility, enzyme activity and oligomer structure of K304Q MCAD with or without GroESL co-overexpression. (A) JM109 cells cotransformed with the K304Q MCAD plasmid and pCaP ( - GroE) or pGroESL ( + GroE) were grown and induced for the time intervals indicated. Cells were disrupted, centrifuged and the pellet (p) and supernatant (s) fractions subjected to SDS-PAGE followed by Western blotting. MCAD: co-electorophoresed purified MCAD. (B) MCAD activity in the extracts was measured with the product formation assay. MCAD activity is given as $\mu$ mol hydroxy-octanoic acid formed per mg protein and minute $\times 10^{-3}$. (C) Superose 12 sizing chromatography of the supernatants after $3 \mathrm{~h}$ induction without ( - GroE) and with (+GroE) GroESL co-overexpression. Aliquots of the fractions (à $0.5 \mathrm{ml}$ ) were analyzed by Western blotting using anti-MCAD antibodies. 
analyzed by Western blotting. The amount of protein loaded and the conditions for Western blotting and chemiluminescence development were identical in order to be able to make semiquantitative comparisons. The results are depicted in Fig. 2. Wild-type MCAD elutes according to the tetramer molecular weight. In the samples with GroESL co-overexpression a small proportion elutes as high molecular weight species. These MCAD molecules are probably associated with GroEL, because GroEL protein was detected in the same fractions with an anti-GroEL antibody (data not shown).

K304E MCAD co-overexpressed with GroESL gives rise to the same two peaks. However, the relative proportion of tetrameric mutant protein is much lower than that of the wild-type protein. If it is taken into consideration, that an amount of soluble K304E MCAD comparable to that of the wild-type protein is present in the supernatant loaded on the column, apparently only a minor amount of $\mathrm{K} 304 \mathrm{E}$ MCAD protein is recovered in the fractions. Without GroESL co-overexpression no tetrameric and no high molecular weight MCAD are detectable under these conditions.

MCAD activity in the fractions from the column run with K304E co-overexpressed with GroESL was measured and the blot of the fractions scanned. As depicted in Fig. 2B, MCAD activity can only be detected in the fractions corresponding to the tetramer molecular weight and not in the high molecular weight species. This corroborates the notion that the high molecular weight species is non-functional incompletetely folded/assembled MCAD. The sum of the activity values comprise about $70 \%$ of the absolute activity in the extract loaded. Therefore, the deficit in recovery stressed above is largely due to loss of inactive, probably incompletely folded/assembled MCAD.

Consequences of an artificial lysine to glutamine mutation at position 304 on MCAD properties

In order to investigate whether the positive charge of lysine-304 is crucial for correct folding and oligomer assembly of the mutant protein, we constructed a mutant in which lysine-304 was exchanged with glutamine by site-directed mutagenesis. Glutamine is very similar to lysine and glutamic acid in steric properties, hydrophobicity and Chou-Fasman helix probability but carries no charge in the side chain at physiological $\mathrm{pH}$. The K304Q mutant was expressed in absence or presence of co-overexpressed GroESL. Fig. 3 summarizes the results obtained with this mutant. The amount of soluble K304Q MCAD is lower without GroESL cooverexpression than with GroESL co-overexpression (Fig. 3A). In the $3 \mathrm{~h}$ induction sample without GroESL co-overexpression a marked degradation band can be observed in the pellet fraction. Such a pronounced degradation band is not observed with GroESL cooverexpression and was also not present in corresponding samples of the wild-type and K304E mutant (see Fig. 1A). Interestingly, detectable quantities of K304Q MCAD protein can be detected at $t=0$ already, while this was not the case in the induction experiments with the wild-type and K304E mutant MCAD.

The effect of GroESL co-overexpression on the partition into the soluble and insoluble fractions is

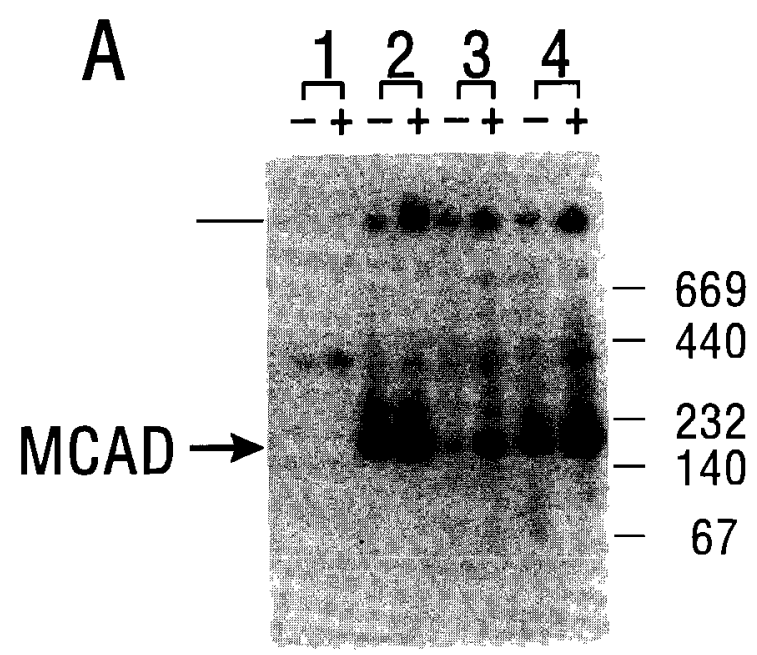

B
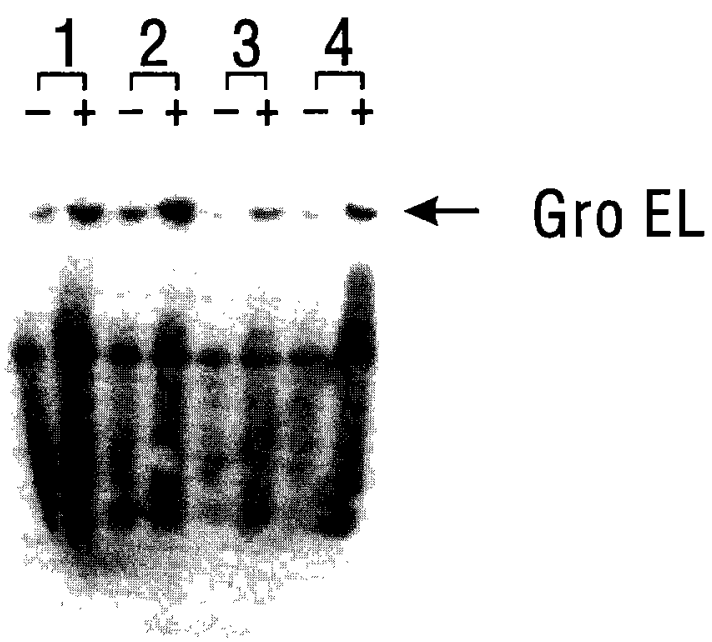

Fig. 4. Native gel electrophoresis. Samples from the extracts after $3 \mathrm{~h}$ induction from the experiments shown in Figs. 1 and 3 were run on a 4-15\% native PAGE gel as described in Materials and Methods. Cells cotransformed with the wild-type (2), K304E (3), or K304Q (4) MCAD plasmids or a control plasmid (1) without $(-)$ or with $(+)$ GroESL co-overexpression were analyzed. The gels were blotted and immunologically developed with anti-MCAD antibody (A) and anti-GroEL antibody (B). The positions and molecular mass in kDa of co-electrophoresed marker proteins are indicated at the right margin of gel A. The arrows show the positions of tetrameric MCAD and the GroEL tetradecamer. Numerical values for MCAD enzyme activity of the respective extracts ( $\mu \mathrm{mol} / \mathrm{mg}$ min; compare Figs. $1 \mathrm{~B}$ and $3 \mathrm{~B}$ ) were: lane $1(-)$ : $0.2:$ lane $1(+): 0.1:$ lane $2(-): 59.0 ;$ lane $2(+): 71.0:$ lane $3(-): 0.7 ;$ lane $3(+): 4.2 ;$ lane $4(-): 12.1$; lane $4(+): 71.0$. 
similar to that obtained for the K304E mutant. However, considerable activity can be measured without GroESL co-overexpression and values comparable to the wild-type level are observed with GroESL co-overexpression. There is an approx. 6-fold increase in yield of active enzyme with GroESL co-overproduction. Interestingly, the values after $25 \mathrm{~h}$ induction are higher than after $3 \mathrm{~h}$ (Fig. 3B) and the $25 \mathrm{~h}$ value is about 2 times as high as the highest relative activity in the wild-type samples. This might be an indication that this mutant is more stable than the wild-type protein.

The results obtained with the activity determination are in line with the data from gel filtration chromatography of the $3 \mathrm{~h}$ induction samples. Without GroESL co-overexpression a small amount of tetrameric K304Q $\mathrm{MCAD}$ is detected upon gel filtration chromatography (Fig. 3C). With GroESL, co-overexpression the picture resembles that obtained for the wild-type protein with GroESL co-overproduction: some high molecular weight material and a large amount of tetrameric $\mathrm{K} 304 \mathrm{Q}$ protein.

\section{Native PAGE analysis}

Native PAGE of the supernatants from cells expressing wild-type, K304E or K304Q MCAD with and without co-overexpression of GroESL was performed to substantiate association of MCAD with GroEL and to compare the amounts of the different forms of MCAD protein present in the supernatants. For this purpose, the extracts after $3 \mathrm{~h}$ induction were run in duplicate on two 4-15\% gradient gels, blotted and immunologically developed with anti-MCAD and antiGroEL antibodies, respectively. The blots are shown in Fig. 4. MCAD protein with an apparent molecular weight corresponding to the tetramer can be detected in all MCAD expressing samples. The intensities of

\section{TABLE I}

Specific activity of wild-type and K304E and K304Q mutant MCAD enzymes

Concentration of purified wild-type and K304Q MCAD were determined with the Bio-Rad protein assay and the concentration of $\mathrm{K} 304 \mathrm{E}$ was calculated from scanning of a Western blot with enriched K304E MCAD and serial dilutions of purified wild-type MCAD. MCAD activity was measured with the mass-spectrometric assay (panel A) and the ferricenium assay (panel B) as described in Materials and Methods. The mean values \pm S.D. and the number of assays (in parentheses) are given.

\begin{tabular}{lll}
\hline MCAD & Specific activity & \\
\cline { 2 - 3 } & $\mathrm{A}$ & $\mathrm{B}$ \\
$\mu$ mol OH-octanoic & $\mu$ mol Ferricenium \\
& acid formed $/$ min $\mathrm{mg}$ & reduced $/$ min $\mathrm{mg}$ \\
\hline $\mathrm{wt}$ & $3.7 \pm 1.2(6)$ & $7.3 \pm 0.9(6)$ \\
$\mathrm{K} 304 \mathrm{E}$ & $1.8 \pm 0.8(4)$ & $6.0 \pm 0.1(2)$ \\
$\mathrm{K} 304 \mathrm{Q}$ & $1.9 \pm 1.2(6)$ & $4.1 \pm 0.8(6)$ \\
\hline
\end{tabular}

this band correspond roughly to the relative amounts of MCAD activty measured in the extracts (numeric values are given in the legend to Fig. 4).

There is a high molecular weight band which is stained both with the MCAD antibody and the GroEL antibody. This band lights up upon GroESL co-overexpression. No band at this position is observed in the control samples developed with the MCAD antibody (A, panel 1). This strongly corroborates that MCAD is transiently associated with GroEL during folding. Also in this system, no monomeric or dimeric K304E MCAD can be detected and there is also no dramatic increase in the quantity of $\mathrm{K} 304 \mathrm{E} \mathrm{MCAD}$ protein bound to GroEL. Comparing the respective amounts of wild-type and K304E MCAD detected in SDS-PAGE (Fig. 1A) and native PAGE, one must conclude that a considerable amount of the mutant protein is not detected in native PAGE.

\section{Specific activity}

In a first attempt to determine the specific activity of the enzymes, wild-type, K304E and K304Q mutant MCAD co-overexpressed with GroESL were purified by subsequent Matrex blue and gel filtration chromatography. Due to the low amount of tetrameric $\mathrm{K} 304 \mathrm{E}$ mutant protein (approx. 0.1\% of total soluble protein), this protein could only be enriched by this procedure. Therefore, in order to estimate the specific activity of the K304E mutant, SDS-PAGE followed by Western blotting with serial dilutions of purified wildtype and enriched K304E MCAD was performed. The bands were scanned and the concentration of the K304E mutant protein in relation to wild-type MCAD was calculated. MCAD activity of the purified or enriched enzymes was determined both with the massspectrometric assay and the ferricenium assay. The values for the respective wild-type and mutant enzymes are given in Table I. They are clearly in the same range. There is a slight indication, that the mutant enzymes might have a somewhat lower specific activity than the wild-type. From these data we conclude that the specific activity of the correctly folded mutant proteins is not or only to a minor degree affected by the mutations. A thorough investigation of minor effects, however, can only be achieved if considerably higher amounts of the K304E mutant protein are available in pure form.

\section{Discussion}

In the present study, using heterologous expression in $E$. coli, we demonstrate, that the disease-causing K304E MCAD is capable to fold properly and assemble into active tetrameric enzyme. Clearly detectable amounts of active K304E tetramer, however, are only produced in this system, when the chaperonins GroEL 
and GroES are co-overexpressed. Another important finding is, that purified tetrameric K304E and the artificially constructed $\mathrm{K} 304 \mathrm{Q}$ mutant display a specific activity in the range of the wild-type protein indicating that enzyme activity is not or only to a minor extent affected by the mutations at position 304. Taken together, this strongly suggests that the K304E mutation primarily influences the rate of folding and/or subunit assembly rather than the specific activity of the assembled enzyme. This conclusion is in line with the finding that residue 304 is not situated in the vicinity of the active site, but rather at the interface between the subunits and therefore is more likely to influence folding and/or subunit assembly [25].

Fig. 5 gives a simplified model for GroESL assisted folding and oligomer assembly of MCAD in order to illustrate the steps at which the K304E mutation might influence the process as discussed in the following.

The major effect of GroESL co-overproduction observed in our experiments is an increase in the proportion of soluble and a concomitant decrease in the proportion of insoluble K304E MCAD, suggesting that the higher chaperonin level prevents aggregation of the mutant protein. As far as the partition between soluble and insoluble MCAD protein is concerned, the K304Q mutant displays a picture very similar to the K304E mutant both with and without GroESL co-overexpression, respectively. This means, that the exchange of lysine-304 to glutamine, as far as the aggregation tendency is concerned, has a similar effect as the lysine to glutamic acid mutation.

An intriguing result was, that a large proportion of the soluble K304E MCAD protein detected by SDSPAGE/Western blot of the extracts escaped detection both after gel filtration chromatography and native
PAGE. The loss of K304E material is not an artifact of the method as it was reproducibly observed and the same effect could not be seen when the wild-type or K304Q mutant proteins were treated the same way. Strikingly, the pool of active K304E protein was to a large degree recovered as active tetramers in gel filtration. An amount of GroEL-complex comparable to that observed for the wild-type and the $\mathrm{K} 304 \mathrm{Q}$ protein was detected. No monomeric or dimeric K304E MCAD was observed in any of the experiments. Disappearance during analytical processing could be due to proteolytic digestion of improperly folded/assembled, soluble K304E MCAD or sticking to the column and gel matrices upon separation from GroEL.

Considering the yield of active tetramer, one must conclude that the bulk both of soluble wild-type and K304Q MCAD are efficiently converted into active tetrameric enzyme, while only a minor part of the $\mathrm{K} 304 \mathrm{E}$ mutant MCAD is present in the active tetrameric form. As solubility of K304E MCAD is stimulated by elevated GroESL levels, a large part of the improperly folded/assembled soluble pool of K304E protein must have interacted with the chaperonins. This form of K304E MCAD is shown in brackets in Fig. 5 in order to emphasize that its precise nature could not be defined experimentally.

Thus, three different patterns have been observed in our experiments depending on which of the three amino acids - lysine, glutamine or glutamic acid - are present at position 304: (i) good solubility and corresponding formation of active tetramer largely independent from GroESL co-overexpression for the wild-type protein; (ii) decreased solubility for the K304Q mutant responsive to GroESL co-overexpression, but good correlation between solubility and amount of active tetramer;

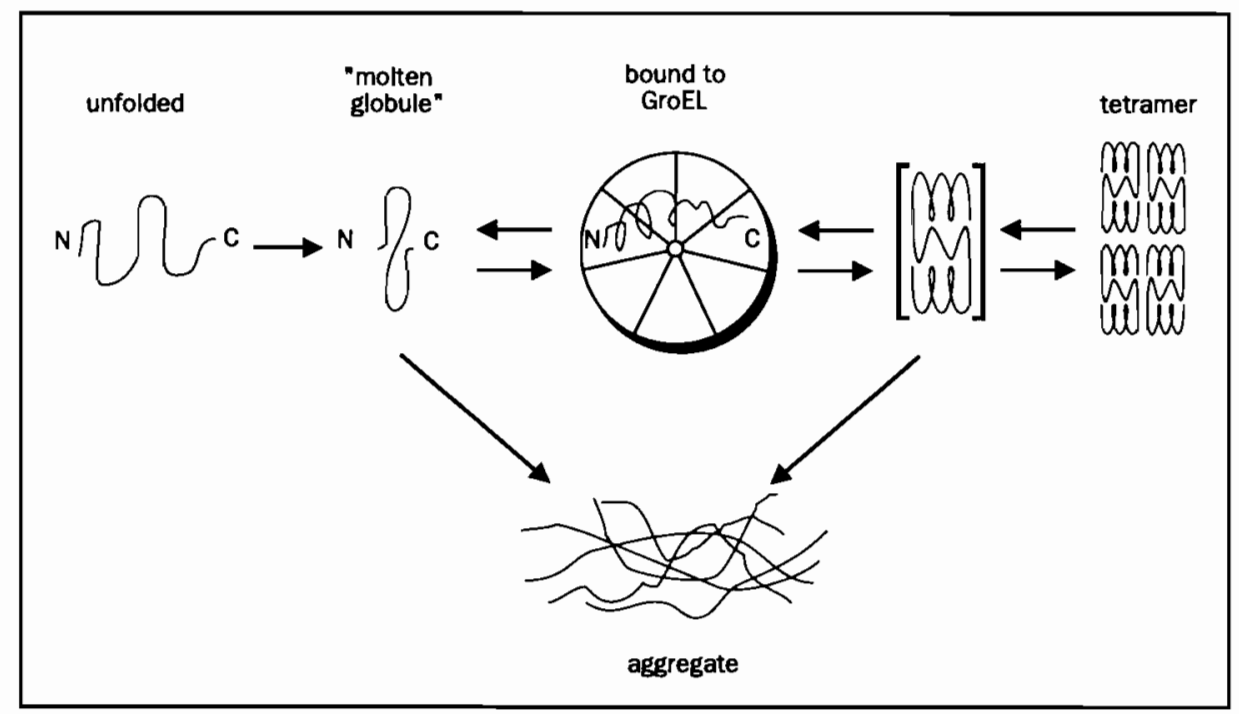

Fig. 5. Model for chaperonin assisted folding and oligomer assembly of MCAD. For details see text. 
(iii) GroESL responsive solubility but non-correlation between solubility and amount of active tetramers for the K304E mutant.

Based on these data, we propose that the K304E mutation has a 2-fold effect: one on the folding pathway and another on the efficiency of subunit assembly. For the folding pathway, the presence of a lysine side-chain at position 304 appears to be essential, as substitution of the negatively charged glutamic acid (K304E MCAD) by the K304Q mutation does not alter the aggregation tendency. A larger pool of GroESL counteracts aggregation by interacting with folding intermediates which have a tendency to self aggregate. A theoretical model for chaperonin action in accordance with such an interpretation has been proposed recently [26]. Pointmutations which "alter the folding pathway without influencing the properties of the native form" have been identified and carefully characterized for the tailspike protein of bacteriophage P22 and suppressor pointmutations in the same protein could be selected which overcome the defect [27]. It has also been shown, that GroESL co-overproduction can suppress point mutations in several diverse bacterial genes [28].

The second effect appears to operate at a later step in folding and subunit assembly. We observed a considerably high amount of non-native K304E MCAD in the soluble fraction which escaped detection under nondenaturing conditions. This was not the case for the wild-type and $\mathrm{K} 304 \mathrm{Q}$ proteins. A possible explanation for this phenomenon can be derived from the X-ray structure data of pig liver MCAD [25]: lysine-304 forms a hydrogen bond to glutamine-342, however, this hydrogen bond can also be formed by a glutamic acid residue at position 304 if it is modelled in. More importantly, the presence of two negative charges, glutamic acid-300 and aspartic acid-346 in a distance of about $6 \AA$ from lysine-304 results in a very high concentration of negative charges at the interface between the subunits if the positively charged lysine-304 is mutated to the negatively charged glutamic acid. This might lead to to impaired subunit assembly due to a charge repulsion effect. This could lead to accumulation of the labile form which escaped detection. A more thorough investigation of this aspect might be possible with an in vitro refolding approach.

The approach with co-overexpression of GroESL is a useful assay system to investigate whether a given mutation influences folding and/or oligomer assembly. Besides the prevalent K304E mutation, seven rare point mutations have been identified in MCAD deficiency so far (Refs. 29 and 30, and B. Andresen, pers. communication). None of these are directly participating in the active site, and are therefore also likely to influence folding and oligomer assembly. This will be addressed experimentally.

The question is to what degree the findings reported with the E. coli expression system are applicable to the situation in eucaryotic mitochondria. Expression of the mature part of the protein in E. coli mimics the situation in mitochondria, where the mature unfolded protein is released into the matrix space and subsequently folds and assembles into tetramers. The chaperone assisted folding process is considered to proceed in the same way in the procaryotic cytoplasm and mitochondria and analogous components, some of which are exchangeable in in vitro assays, are involved. However, both in patient fibroblasts and in COS-7 cell expression of $\mathrm{K} 304 \mathrm{E}$ mutant MCAD no aggregates have been identified, but rather a lower stability of the MCAD polypeptide as monitored by SDS-PAGE has been observed. On the other hand, instability of tetrameric K304E mutant MCAD was not indicated in $E$. coli. The main difference, however, could be the different ways incompletely folded/assembled proteins are handled in mitochondria and E. coli. It is likely, that also in mitochondria, K304E mutant folding intermediates accumulate and that these rather than assembled tetramers represent the species that is degraded. Thus, also in the eucaryotic cell, probably only a small proportion of the mutant protein acquires the native structure and MCAD activity levels are low mainly due to this phenomenon.

\section{Acknowledgements}

We thank Drs. Jung-Ja Kim and Jens Birktoft for helpful and valuable discussions concerning MCAD structure. We are indebted to Anthony Gatenby for providing the pGroESL plasmid and anti-GroEL antibodies. We thank Eigil Kjeldsen for help with the scanning measurements. P.B. was supported by a bursary from the SCIENCE program of the Commission of the European Communities.

\section{References}

1 Beinert, H. (1963) The Enzymes 7, 447-476.

2 Ghisla, S., Engst, S., Inagaki, T., Ohishi, N. and Yagi, K. (1991) in New Trends in Biological Chemistry (Ozawa, T., ed.), pp. 1-19, Japan Sci. Soc. Press, Tokyo.

3 Izai, K., Uchida, Y., Orii, T., Yamamoto, S. and Hashimoto, T. (1992) J. Biol. Chem. 267, 1027-1033.

4 Anonymous (1992) in New developments in fatty acid oxidation (Coates, P.M. and Tanaka, K., eds.), pp. 499-506, John Wiley and Sons, New York.

5 Gregersen, N., Andresen, B.S., Bross, P., Winter, V., Engst, S., Rüdiger, N., Christensen, E., Kelly, D., Strauss, A.W., Kølvraa, S., Bolund, L. and Ghisla, S. (1991) Hum. Genet. 86, 545-551.

6 Matsubara, Y., Narisawa, K., Miyabayashi, S., Tada, K., Coates, P.M., Bachmann, C., Elsas, L.J., Pollitt, R.J., Rhead, W.J. and Roe, C.R. (1990) Biochem. Biophys. Res. Commun. 171, 498-505.

7 Yokota, I., Indo, Y., Coates, P.M. and Tanaka, K. (1990) J. Clin. Invest. 86, 1000-1003.

8 Kelly, D.P., Whelan, A.J., Ogden, M.L., Alpers, R., Zhang, Z., Bellus, G., Gregersen, N., Dorland, L. and Strauss, A.W. (1990) Proc. Natl. Acad. Sci. USA 97, 9326-9240. 
9 Ikeda, Y., Keese, S.M., Fenton, W.A. and Tanaka, K. (1987) Arch. Biochem. Biophys. 252, 662-674.

10 Bross, P., Jensen, T.G., Kraeutle, F., Winter, V., Andresen, B.S., Engst, S., Bolund, L., Kølvraa, S., Ghisla, S., Rasched, I. and Gregersen, N. (1992) in New developments in fatty acid oxidation (Coates, P.M. and Tanaka, K., eds.), pp. 472-478, John Wiley and Sons, New York.

11 Jensen, T.G., Andresen, B.S., Bross, P., Jensen, U.B., Holme, I., Kølvraa, S., Gregersen, N. and Bolund, L. (1992) Biochim. Biophys. Acta 1180, 65-72.

12 Coates, P.M., Indo, Y., Young, D., Hale, D.E. and Tanaka, K. (1992) Pediatr. Res. 31, 34-38.

13 Gatenby, A.A. (1992) Plant. Molec. Biol. 19, 677-687.

14 Hartl, F.U., Martin, J. and Neupert, W. (1992) Ann. Rev. Biophys. Biomol. Struct. 21, 293-322.

15 Goloubinoff, P., Gatenby, A.A. and Lorimer, G.H. (1989) Nature $337,44-47$.

16 Hemmingsen, S.M., Woolford, C., van der Vies, S.M., Tilly, K., Dennis, D.T., Georgopoulos, C.P., Hendrix, R.W. and Ellis, R.J. (1988) Nature 333, 330-334.

17 Yanisch-Perron, C., Vieira, J. and Messing, J. (1985) Gene 33, 103-119.

18 Miller, J.H. (1972) Experiments in Molecular Genetics, Cold Spring Harbor, New York.
19 Kølvraa, S., Gregersen, N., Christensen, E. and Hobolth, N. (1982) Clin. Chim. Acta 126, 53-67.

20 Lehman, T.C., Hale, D.E., Bhala, A. and Thorpe, C. (1990) Anal. Biochem. 186, 280-284.

21 Kuipers, O.P., Boot, H.J. and DeVos, W.M. (1991) Nucl. Acids Res. 19, 4558.

22 Laemmli, U. (1970) Nature, 227, 680-685.

23 Blake, M.S., Johnsten, G.J., Russel-Jones, G.J. and Gotschlich, E.C. (1984) Anal. Biochem. 136, 175-179.

24 Mitraki, A. and King, J. (1989) Bio/Technol. 7, 690-697.

25 Kim, J.-J.P., Wang, M., Djordjevic, S. and Paschke, R. (1992) in New developments in fatty acid oxidation (Coates, P.M. and Tanaka, K., eds.), pp. 111-126, John Wiley and Sons, New York.

26 Roy, H., Kupferschmid, M. and Bell, J.A. (1992) Protein Science 1, 925-934.

27 Mitraki, A. and King, J. (1992) FEBS Lett. 307, 20-25.

28 Van Dyk, T.K., Gatenby, A.A. and LaRossa, R.A. (1989) Nature $342,451-453$.

29 Yokota, I., Coates, P.M., Hale, D.E., Rinaldo, P. and Tanaka, K. (1991) Am. J. Hum. Genet. 47, 1280-1291.

30 Andresen, B.S., Bross, P., Winter, V., Kølvraa, S., Jensen, T.G., Bolund, L., Duran, M., Kim, J.-J., Curtis, D., Divry, P., VianeySaban, C. and Gregersen, N. (1993) Am. J. Hum. Genet., in press. 